\title{
Alain Chatriot, Odile Join-Lambert, et Vincent Viet, Les politiques du Travail (1906-2006) : acteurs, institutions, réseaux
}

\section{Herrick Chapman}

\section{(2) OpenEdition}

\section{Journals}

Édition électronique

URL : http://journals.openedition.org/travailemploi/4606

DOI : $10.4000 /$ travailemploi.4606

ISSN : 1775-416X

Éditeur

DARES - Ministère du Travail

Édition imprimée

Date de publication : 30 mars 2007

ISSN : 0224-4365

Référence électronique

Herrick Chapman, « Alain Chatriot, Odile Join-Lambert, et Vincent Viet, Les politiques du Travail (1906-2006) : acteurs, institutions, réseaux », Travail et Emploi [En ligne], 110 | Avril-Juin 2007, mis en ligne le 20 décembre 2012, consulté le 22 septembre 2020. URL : http://journals.openedition.org/ travailemploi/4606 ; DOI : https://doi.org/10.4000/travailemploi.4606 


\section{Les politiques du Travail (1906- 2006): acteurs, institutions, réseaux}

\author{
Alain Chatriot, Odile Join-Lambert, \\ et Vincent Viet (sous la direction de)
}

\author{
Rennes, Presses Universitaires de Rennes, \\ 2006, 518 pages.
}

\section{Lu par Herrick Chapman, New York University}

Ce volume remarquable est issu d'un colloque international qui a eu lieu en mai 2006 à l'occasion du centenaire de la création du ministère du Travail en France. Il a pour but d'examiner un siècle d'intervention étatique dans le monde du travail. Le livre rassemble vingt-sept articles, une excellente introduction de la part des éditeurs qui situe le travail au sein d'une plus large réflexion sur l'histoire de l'État, ainsi que huit brefs témoignages de hauts fonctionnaires et professeurs associés au ministère du Travail. Ce qui émerge de la collection n'est pas une fresque cohérente représentant l'histoire du ministère, mais quelque chose de mieux : un collage pluridisciplinaire de nouvelles recherches et de témoignages qui élargissent notre compréhension des mécanismes internes au ministère, ses rapports aux autres institutions, et son rôle dans de vastes transformations historiques qui ont fait de ce $\mathrm{XX}^{\mathrm{e}}$ siècle violent une étonnante époque pour l'expansion de l'État.

Plusieurs des essais, qui se concentrent sur la période entre 1890 et 1914, révèlent que les origines du ministère viennent non seulement par en haut, mais aussi par en bas. Le ministère est d'une certaine manière une invention élite des réformateurs tels qu'Arthur Fontaine qui s'intègrent au gouvernement grâce au ministère du Commerce d'Alexandre Millerand. Les relations internationales jouent aussi un rôle dans le développement du ministère du Travail. L'autorité de ce dernier dans les affaires d'immigration, par exemple, comme le montrent Caroline Douki, David Feldman et PaulAndré Rosental, naît des négociations bilatérales en 1904 entre la France et l'Italie consacrées à l'élargissement de la protection sociale des migrants dans leurs pays respectifs. Néanmoins, selon Françoise Bosman, Claude Chetcuti et Jean-François Eck, l'établissement de l'inspection du travail en 1892 et du ministère du Travail en 1906 doit beaucoup à des expérimentations au niveau local dans le domaine des inspections et des réglementations de sécurité, particulièrement dans le Nord et le Pasde-Calais. Philippe Hamman dépeint la Lorraine pendant l'occupation comme une sorte de laboratoire pour la protection sociale qui influencera plus tard la politique nationale. Des changements subtils et diffus de mentalité accompagnent l'émergence du nouveau ministère. Pierre Vargus démontre, par exemple, comment un patronat lyonnais ultraconservateur s'accommode du nouveau ministère parce qu'il s'est trouvé obligé de s'adapter aux nouvelles formes d'inspection du travail et de régulations étatiques dans les années 1890. Jacques Le Goff soutient aussi que les dirigeants républicains apprennent à modérer leur Jacobinisme juste assez pour chercher des liens plus forts entre l'État et les organismes ouvriers via le paritarisme au sein du Conseil supérieur du travail et des commissions départementales $\mathrm{du}$ travail. Les syndicalistes militants, eux aussi, malgré leur rhétorique anti-étatique, prennent un intérêt pragmatique à une plus grande présence régulatrice de l'État dans leur lieu de travail. En 1914, le ministère est dorénavant légitimé et possède les outils principaux pour toute intervention sociale - inspections, enquêtes, tripartisme - des outils que, comme le montre Isabelle Lespinet Moret, Arthur Fontaine transplantera à l'OIT à Genève dans les années 1920 pour donner à cette institution une personnalité française.

Si les premières années du ministère sont cruciales, il faut aussi tenir compte du rôle des deux guerres mondiales dans son évolution. Vincent Viet soutient qu'à la fin de la Première Guerre mondiale, le ministère hérite de la part du ministère de l'Armement de nouvelles responsabilités pour les politiques de maind'œuvre, ce que, dans les décennies qui suivent, le ministère ne parviendra jamais à bien intégrer dans sa mission fondamentale, celle de l'inspection et de la régulation des contrats de travail. L'inspection et la main-d'œuvre resteront deux domaines distincts de la politique et du droit. La Deuxième Guerre mondiale, ainsi que la reconstruction, ne font que perpétuer le problème. La Deuxième Guerre mondiale compromet aussi l'intégrité du ministère en l'impliquant dans la relève et le STO. Dans son étude de l'épuration, Jean-Pierre Le Crom montre que plusieurs inspecteurs supportent mal ces politiques et que l'épuration d'après-guerre semble uniquement cibler les cas les plus flagrants de collaboration idéologique. Le processus permet néanmoins au ministre Alexandre Parodi de contourner les règlements concernant l'ancienneté pour placer de nouveaux leaders dynamiques dans de hauts postes.

La guerre et la Libération inaugurent aussi un effort impressionnant visant à institutionnaliser la démocratie industrielle du $\mathrm{XX}^{\mathrm{e}}$ siècle français à travers les comités d'entreprise, les comités d'hygiène et de sécurité, les délégués du personnel, et les caisses de la Sécurité sociale. Ces organismes accordent au ministère un rôle majeur dans la démocratisation de l'après-guerre. Selon Alain Chatriot, la capacité du ministère de jouer ce rôle se voit dans son implication pendant l'entre-deux-guerres dans la formation du Conseil national économique. Par contraste, Orjan Appleqvist, Adam Steinhouse 
et Nicolas Hatzfeld prétendent que c'est plutôt la rupture de la Libération, ainsi que l'insurrection des syndicats et de la gauche en 1944-1946, qui a rendu possible ces innovations. Ils soulignent aussi à quel point cette expérimentation de la démocratie industrielle est écourtée après 1946, lorsque l'élite politique de la IVe République choisit de poursuivre la reconstruction et la modernisation en partenariat avec le patronat à l'exclusion d'une grande partie du mouvement ouvrier. La politique de la guerre froide contribue évidemment à ce renversement à la bonne fortune du mouvement ouvrier, mais il est aussi la conséquence des liens de longue durée que le patronat a cultivés avec l'État, y compris le ministère du Travail d'avant la guerre, un sujet qu'explore Danièle Fraboulet dans sa contribution sur l'UIMM et le ministère. Les forces du patronat et les faiblesses du mouvement ouvrier resteront une caractéristique centrale du paysage social après 1950 - un arrière-plan essentiel pour apprécier la contribution de Lucie Tanguy sur l'institutionnalisation de la formation syndicale dans les années 1950 et 1960. Cette institutionnalisation est par ailleurs un processus dans lequel le ministère du Travail joue un rôle étonnamment important pour restreindre le radicalisme du mouvement ouvrier. En outre, Michèle Dupré, Olivier Giraud, Michèle Tallard et Catherine Vincent, dans leurs études sur la démocratie industrielle en France et en Allemagne de l'Ouest de l'après-guerre, reconnaissent à quel point il est difficile pour les ouvriers français de contrer le pouvoir fermement enraciné du patronat.

L'histoire d'un ministère peut aussi être racontée à travers la mutation des politiques et des relations interministérielles. Le rôle du ministère du Travail dans l'agriculture est en particulier une source de contestations jusqu' au moment où le régime de Vichy décide que le ministère de l'Agriculture dirigera l'administration des réglementations ouvrières et de la protection sociale dans ce secteur, ce dont parlent Claire-Élise Michard et René Bourrigaud. Le logement social fait aussi partie du portfolio ministériel, du moins jusqu'aux années 1930, lorsque, comme le montre Hélène Frouard, il devient plutôt associé à l'urbanisme. La dissociation du logement social et des principes de la protection sociale, ainsi que la popularité de l'idéal du «citoyen-propriétaire», repoussent d'une génération tout véritable investissement dans le logement social. La relation compliquée que le ministère du Travail entretient avec le ministère de l'Éducation nationale après la Deuxième Guerre mondiale est le sujet de la contribution d'Odile Join-Lambert, qui se penche sur la confusion qui règne à propos de l'autorité et du droit en ce qui concerne les accidents professionnels des élèves de l'enseignement technique entre 1946 et 1982 . Même le rôle du ministère de l'après-guerre dans la politique de la famille et de la Sécurité sociale qui, comme le montre Michel Dreyfus, a toujours été central à la mission du ministère, devient beaucoup plus ambigu aux débuts de la Ve République. À ce moment, comme l'explique Bruno Béthouart, la politique sociale devient à la fois plus directement contrôlée par le Premier ministre, et aussi plus dispersée parmi les différents ministères. De plus, la politique de l'emploi, ainsi qu'en parlent Jaques Freyssinet et Pierre Maclouf, devient dans les années 1950 et 1960 un domaine de politique plus complexe, à la fois plus central aux ambitions modernisatrices de l'État, et administré de façon plus diffuse par le Plan et par presque tous les ministères associés à l'économie. Béthouart a certainement raison lorsqu'il suggère que l'âge d'or du ministère était pendant la IVe République. S'il en est ainsi, on peut se demander si l'autonomie du ministère au sein de l'État vient en partie de la puissance et de la visibilité du mouvement ouvrier qu'il était censé contenir.

Depuis ces toutes premières enquêtes jusqu'à nos jours, le ministère du Travail joue un rôle important dans la production du savoir, l'invention des catégories sociales, et la mise en place des compétences. Sabine Rudischhauser utilise de façon particulièrement intéressante la méthode comparative pour étayer ce thème en explorant comment les supports institutionnels des conventions collectives en Allemagne avant les années 1920 (et en particulier le rôle important des tribunaux et du droit du travail) produisent un corpus plus robuste de textes de science sociale sur les relations sociales et sur le travail qu'en France. Même au sein du ministère lui-même l'institutionnalisation de la recherche statistique se révèle être un long processus difficile. Comme l'exposent Étienne Penissat et Béatrice Touchelay, les réformes ouvrières pendant le Front populaire et la mobilisation économique depuis 1938 donnent finalement au ministère les moyens de surmonter la résistance des employeurs aux statistiques, ce qui constituait une énorme partie du problème. En outre, depuis 1945, le ministère joue un rôle particulièrement important dans l'élaboration des bases de données à propos de l'immigration, comme le montre Laure Pitti dans son étude des revues professionnelles du ministère durant la période 1945-1984. La connaissance, l'information, et la politique resteront toujours étroitement impliquées les unes dans les autres, un fait révélé par les controverses actuelles à propos de la politique antidiscriminatoire et des statistiques d'embauche.

Pourtant, même un livre de cette envergure n'évite pas les lacunes, et invite à de nouvelles recherches. Les éditeurs offrent leur propre agenda ambitieux pour de futures recherches, y compris, par exemple, des études sur les effectifs des services du ministère. La contribution fascinante d'Anne-Sophie Beau et Sylvie Schweitzer sur la longue lutte des inspectrices pour surmonter la discrimination et pour être mieux intégrées dans le travail de l'inspection du Travail montre ce qui peut être révélé par cette sorte d'investigation. De même, plus de spécialistes bénéficie- 
raient de l'exemple de Douki, Feldman et Rosental en explorant comment les liens internationaux et les mutations de l'environnement international changent la structure et le rôle de l'État et de son rapport au monde du travail. Par ailleurs, ce contexte international se doit d'inclure les États-Unis, (qui figurent très peu dans ce volume) et l'Empire français. Le texte de Florence Renucci sur l'application curieuse du Code du travail dans l'AOF, et le témoignage de Jean-Paul Chaze sur sa propre expérience en tant que directeur adjoint en Polynésie, suggèrent l'importance de la France d'outre-mer dans cette histoire. L'oubli le plus surprenant dans ce volume est le manque relatif de nouvelles recherches sur les ouvriers, les syndicats, et leurs rapports au ministère. Nous apprenons plus à propos du patronat qu'à propos du travail. Finalement, l'histoire du ministère et de l'intervention de l'État dans le monde du travail après les Trente Glorieuses ne vient que de commencer. Ici, l'écriture des témoins est particulièrement utile puisque la plupart de leurs observations concernent les défis qu'ils ont affrontés pendant ces trois dernières décennies de croissance lente, désindustrialisation, et haut chômage. Ce volume, donc, servira de catalyseur pour de nouvelles explorations, alors même qu'il est un dépôt extraordinairement riche d'idées, d'observations et de recherches récentes.

\section{La politique sociale en Allemagne 1945-1994 Geschichte der Sozialpolitik in Deutschland seit 1945}

Éditeur: ministère fédéral des Affaires sociales et des Archives fédérales, Bundesministerium für Arbeit et Bundesarchiv, 11 vols

Nomos, 2001-2007.

Band 1: Grundlagen der Sozialpolitik.

Band 2: 1945 - 1949 Die Zeit der Besatzungszonen. Sozialpolitik zwischen Kriegsende und der Gründung zweier deutscher Staaten, hg. v. Prof. Dr. Udo Wengst, 2001.

Band 3: 1949 - 1957 Bundesrepublik Deutschland, Bewältigung der Kriegsfolgen, Rückkehr zur sozialpolitischen Normalität, hg. v. Prof. Dr. Günther Schulz, 2004.

Band 4: 1957 - 1966 Bundesrepublik Deutschland. Sozialpolitik im Zeichen des erreichten Wohlstands, hg.v. Prof. Dr. Michael Ruck, PD Dr. Marcel Boldorf, à paraître.

Band 5: 1966 - 1974 Bundesrepublik Deutschland, Eine Zeit vielfältigen Aufbruchs, hg. v. Prof. Dr. Hans Günter Hockerts, 2007.

Band 6: 1974 - 1982 Bundesrepublik
Deutschland. Neue Herausforderungen, wachsende Unsicherheit, hg. v. Prof. Dr. Martin H. Geyer, 2007.

Band 7: 1982 - 1989 Bundesrepublik Deutschland, Finanzielle Konsolidierung und institutionelle Reform, hg. v. Prof. Dr. Manfred G. Schmidt, 2005.

Band 8: 1949 - 1961 Deutsche Demokratische Republik. Im Zeichen des Aufbaus des Sozialismus, Hg. im Auftrag des Instituts für Zeitgeschichte München_Berlin: Dr. Dierk Hoffmann, PD Dr. Michael Schwartz, 2004.

Band 9: 1961 - 1971 Deutsche Demokratische Republik. Politische Stabilisierung und wirtschaftliche Mobilisierung, hg. v. Prof. Dr. Christoph Kleßmann, 2006.

Band 10: 1971 - 1989 Deutsche Demokratische Republik. Bewegung in der Sozialpolitik, Erstarrung und Niedergang, hg. v. PD Dr. Christoph Boyer, Prof. Dr. Klaus_Dietmar Henke z, Dr. Peter Skyba, 2007.

Band 11: 1989 - 1994 Bundesrepublik Deutschland. Sozialpolitik im Zeichen der Vereinigung, hg.v. Prof. Dr. Gerhard A. Ritter, 2007.

\section{Lu par Rainer Hudemann, universität des Saarlandes}

Par rapport à la France, la situation de la politique sociale allemande en 1945 fut très différente. Ceci non seulement à la suite du partage du pays qui s'amorça d'abord rapidement dans la réalité et ensuite juridiquement. L'Allemagne revêtait en Europe une position de pionnière en politique sociale depuis les années 1880, quand l'État commença à fondre les multiples traditions en un système cohérent d'assurances sociales. Mais ce fut un système dont les structures reflétaient, dans l'organisation et dans les conditions différentes selon les couches sociales et les branches économiques, la société de l'époque de la révolution industrielle. Certains des grands débats actuels en RFA tournent toujours autour de difficultés découlant de cet héritage.

Précisément parce que la France fut plus lente à développer et à mettre en œuvre un système comparable, elle eut en 1944-1946 l'avantage de la modernité: elle avait moins de bagages de traditions et de structures ancrées à surmonter que l'Allemagne. Avec le plan de Sécurité sociale de Pierre Laroque, elle rejoignit l'état international du débat social, parti en 1942 du plan Beveridge et de sa conception d'un système unique d'assurances sociales, intégrées dans une politique générale de sécurité sociale pour toute la société. L'Allemagne fut à un point de rupture fondamental à la fin de la guerre et du régime nazi. Mais les chances qui semblaient 